\title{
Field Assessment of an Immune-Complex Infectious Bursal Disease Vaccine in Chicks Born to Non-Hyperimmunized Broiler Breeders
}

\author{
Cazaban $\mathrm{C}^{\star 1}$, Swart $\mathrm{WBF}^{2}$, Rietema RMW ${ }^{3}$, Wit JJD ${ }^{4}$, Palya $\mathrm{V}^{5}$ and Gardin $\mathrm{Y}^{1}$ \\ ${ }^{1}$ Ceva Animal Health, Libourne, France \\ ${ }^{2}$ Ceva Animal Health Benelux, Naaldwijk, the Netherlands \\ ${ }^{3}$ Pluimveepraktijk Noord \& Oost, Slagharen, the Netherlands \\ ${ }^{4}$ GD Animal Health, Deventer, the Netherlands \\ ${ }^{5}$ Ceva-Phylaxia, Budapest, Hungary
}

*Corresponding author: Cazaban C, Ceva Animal Health, 33500 Libourne, France, E-mail: christophe. cazaban@ceva.com

Citation: Cazaban C, Swart WBF, Rietema RMW, Wit JJD, Palya V, et al. (2018) Field Assessment of An Immune-Complex Infectious Bursal Disease Vaccine in Chicks Born to Non-Hyperimmunized Broiler Breeders. J Vet Sci Ani Husb 6(3): 302. doi: 10.15744/2348-9790.6.302

Received Date: May 29, 2018 Accepted Date: August 21, 2018 Published Date: August 23, 2018

\begin{abstract}
Prevention of infectious bursal disease (IBD) remains of paramount importance, given the dramatic losses it can induce in commercial chicken flocks. In the Netherlands, maternal immunity is regarded as low, because the parent stock flocks do not receive an inactivated vaccination prior to the onset of production. Therefore, the aim of this field study was to assess the consequences of hatchery vaccination against IBD using an immune-complex vaccine in broiler chicks that were provided with a limited amount of passive immunity. Three grow out cycles in two houses each (six flocks) were monitored after vaccination. The recorded parameters included (i) body and bursa weight, (ii) IBD antibody response, and (iii) histopathology and PCR from the bursa of Fabricius. Average growth was similar in all groups, although inferior to the breed's standards. Average bursa weight faced some reduction as part of vaccine effect. Histopathology analysis of the bursa showed a transient inflammatory process, while PCR detected the vaccine strain only. Lastly, serology tests confirmed the successful immunization of all broiler flocks. The two commercial ELISA kits (Idexx and BioChek) failed to detect antibodies for a limited period of time, whereas virus neutralization (VN) test could detect antibodies for the entire broilers' lifespan. Idexx and BioChek ELISA kits had the highest correlation coefficient (0.93), whereas the lowest correlation was found between Idexx ELISA and VN (0.68). BioChek ELISA and VN showed a correlation of 0.78. This is the first detailed investigation of immune-complex vaccine's mechanism of action in field conditions in chicks with low maternal immunity at hatch $(<4000)$ and using a comprehensive set of monitoring parameters. It also provides updated information about IBD serology tests.
\end{abstract}

Keywords: Infectious Bursal Disease; Immune-complex Vaccine; Broiler; Serology; ELISA

List of abbreviations: BBR: Bursa To Body Weight Ratio; BFW: Bursa of Fabricius Weight;BW: Body Weight; CEF : Chicken Embryo Fibroblasts ; CV: Coefficient of Variation; DOC: Day-old Chick(s); ELISA: Enzyme-Linked Immunosorbent Assay; GLM: Generalized Linear Model; HVT: Herpesvirus of Turkeys; IBD: infectious Bursal Disease; IBDV: Infectious Bursal Disease Virus; MDA: Maternally Derived Antibodies; ND: Newcastle Disease; PS: Parent Stock; RFLP: Restriction Fragment Length Polymorphism; SPF: Specific Pathogen Free; TCID $_{50}$ : Tissue Culture Infectious Dose 50\% ; VN: Virus Neutralization; VNT: Virus Neutralization Test; VP: Viral Proteins (1 to 5); vvIBDV: Very Virulent Infectious Bursal Disease Virus

\section{Introduction}

Infectious bursal disease (IBD) is a viral disease that affects chickens worldwide and it has a major economic impact on the modern poultry industry. It is caused by a birnavirus, called infectious bursal disease virus (IBDV), which is a bi-segmented, double stranded RNA virus, highly resistant in the environment. The RNA encodes for five viral proteins (VP1 to VP5) of which VP2 contains hypervariable portions that enable classifying strains into various antigenic and genetic groups; in addition, VP2 contains the majority of neutralizing sites. Depending on the pathogenic type, IBDV strains are classified as very virulent, virulent, and subclinical. Antigenically two serotypes (serotype 1 and 2) of IBDV exist, although antigenic variants are described within serotype 1 strains. Only the serotype 1 virus is pathogenic for chickens in which it can cause depression, diarrhoea, haemorrhages in muscles and proventriculus, and inflammation, necrosis and atrophy of the bursa of Fabricius depending on the virulence of the strain. Variant IBDV strains generally do not cause clinical signs or mortality, but can cause severe atrophy of the bursa without marked 
inflammatory reaction during the acute stage of the disease. Depending on the age of the birds at infection and their immune status, IBDV can also induce immunosuppression [1]. Recent reports confirmed IBD presence in various parts of the world, namely South America, North America, Africa, China, the Middle East, and Europe [2-10]. Therefore, IBD is prevalent worldwide and its control is a global concern. Biosecurity is key in preventing IBD. However, vaccination is regarded as an important tool to complement biosecurity efforts. Parent stock vaccination is useful to elicit humoral immunity that will be transmitted to the progeny (passive immunity); it will protect the young chicks for the first few weeks of life [11]. Live vaccination is applied in the progeny because passive immunity does not last long enough to ensure adequate protection for the whole broilers' lifespan. By doing so, chickens develop an active immunity. Several vaccines are commercially available for the control of IBD in broilers. Historically, vaccination has been applied in the farm via drinking water using live attenuated vaccines of various residual pathogenicity (mild, intermediate, intermediate plus, hot). Hatchery vaccination is becoming a growing trend because of the increasing capacity of poultry producing companies, a higher degree of integration, and the willingness to better master the vaccine application using automated equipment, either by day-old injection via subcutaneous route, or in ovo route. High-throughput automated equipment is now available (in ovo or day-old injection) enabling a higher quality, consistency, and cost-effectiveness of vaccination [12]. In addition, hatchery vaccination removes the risk of vaccination failure in the field (due to poor water quality, inappropriate timing, and insufficient flock coverage); it also prevents the stress of water withdrawal in the farm when using the classical drinking water method. As a consequence, moving vaccination in the hatchery improves the welfare of the birds in the farm. Altogether, it offers a significant improvement in terms of immunization success as recently demonstrated [13]. Advances in technology have enabled the development of new vaccines that are able to escape the neutralizing effect of maternally derived antibodies (MDA) and hence are eligible for hatchery application. Two types of IBD vaccines are currently available for hatchery application in Europe: (i) an immune-complex vaccine in which a live attenuated IBD vaccine strain has been in vitro complexed with a high titre polyclonal serum in specific quantities; (ii) and, alternatively, a vaccine using the herpesvirus of turkeys (HVT) vector technology as a carrier expressing IBDV's VP2 antigen [14,15].

This field study was performed in the Netherlands, where IBD risk is perceived as minimal. Therefore, breeders do not routinely receive any inactivated $I B D$ vaccine prior to the onset of production, contrary to a hyperimmunization program (using live priming and inactivated booster vaccinations) that would be applied in other countries. As a consequence, the quantity of passively transferred immunity from the breeder hens to the progeny is usually low. This trial was implemented to address some safety concerns: day-old chicks (DOC) with low levels of maternally-derived antibodies (MDA) at hatch could be vaccinated with the immune-complex IBD vaccine. In such case, the release of the IBD vaccine virus would take place early in the chick's life and could, theoretically, impair the immune functions of the bursa of Fabricius, its target organ. To address this issue, hatching eggs coming from non-hyper-immunized parent stock flocks were selected, vaccinated using an immune-complex IBD vaccine and monitored during rearing until harvest using an extensive range of parameters.

\section{Materials and Methods}

\section{Chickens}

Embryonated eggs of Ross 308 genetic line were injected in ovo with one dose of Cevac ${ }^{\circledast}$ Transmune (Ceva Animal Health, Libourne, France) IBD immune-complex vaccine at 18.5 days of embryonic development using Egginject ${ }^{\oplus}$ in ovo injector (Ceva Animal Health, Ecat-ID Campus, Landivisiau, France) according to the manufacturer's instructions. 198,400 broiler chicks which hatched from the vaccinated eggs were included in the trial. The rest of the vaccination and medication scheme during the rearing phase of the chickens was done according to the routinely applied program: it included live vaccination against infectious bronchitis and Newcastle disease, and in-feed medication against coccidiosis. The farm where the study took place followed the "all in-all out" management system. The feed was purchased from the same feed mill throughout the study. It was distributed according to routine practices. Water was provided ad libitum. The broiler farm included ten houses which were of similar construction, structure and equipment. Only two houses out of ten were monitored in this trial. For consistency and repeatability of results, six vaccinated broiler flocks were monitored (three grow-out cycles, two buildings). The first grow-out cycle in two houses (abbreviated 1-1 and 1-2) included 32,800 chickens per house; they were born to a 32-week old parent stock flock. The second grow-out cycle (abbreviated 2-1 and 2-2) included 33,600 chickens per house; they were born to a 42-week old parent stock flock. The third growout cycle (abbreviated 3-1 and 3-2) included 32,800 chickens per house; they were born to a 44-week old parent stock flock. All were reared without sex sorting.

\section{Samplings}

Ten chickens from each house were randomly selected and were transported alive to a local laboratory (Pluimveepraktijk Noord \& Oost, Slagharen, the Netherlands) for weighing and for blood and bursa sampling. Due to organizational constraints, the samplings could not be scheduled similarly in the three cycles. Table 1 summarizes the actual samplings schedule per grow-out cycle according to age. 


\begin{tabular}{|c|c|c|c|c|}
\hline Age (days) & BW & BFW & Serology & Histology \& PCR \\
\hline 7 & $1-1,1-2$ & $1-1,1-2$ & - & $1-1,1-2$ \\
\hline 10 & $1-1,1-2$ & $1-1,1-2$ & $1-1$ & $1-1,1-2$ \\
\hline 11 & - & - & $1-2$ & - \\
\hline 12 & $3-1,3-2$ & $3-1,3-2$ & $3-1,3-2$ & $3-1,3-2$ \\
\hline 14 & $1-1,1-2$ & $1-1,1-2$ & $1-1,1-2$ & $1-1,1-2$ \\
\hline 15 & $2-1,2-2$ & $2-1,2-2$ & $2-1,2-2$ & $2-1,2-2$ \\
\hline 16 & $3-1,3-2$ & $3-1,3-2$ & $3-1,3-2$ & $3-1,3-2$ \\
\hline 17 & $1-1,1-2$ & $1-1,1-2$ & $1-1,1-2$ & $1-1,1-2$ \\
\hline 18 & $3-1,3-2$ & $3-1,3-2$ & $3-1,3-2$ & $3-1,3-2$ \\
\hline 19 & $2-1,2-2$ & $2-1,2-2$ & $2-1,2-2$ & $2-1,2-2$ \\
\hline 21 & $1-1,1-2$ & $1-1,1-2$ & $1-1,1-2$ & $1-1,1-2$ \\
\hline 22 & $2-1,2-2$ & $2-1,2-2$ & $2-1,2-2$ & $2-1,2-2$ \\
\hline 23 & $3-1,3-2$ & $3-1,3-2$ & $3-1,3-2$ & $3-1,3-2$ \\
\hline 24 & $1-1,1-2$ & $1-1,1-2$ & $1-1,1-2$ & $1-1,1-2$ \\
\hline 25 & $3-1,3-2$ & $3-1,3-2$ & $3-1,3-2$ & $3-1,3-2$ \\
\hline 27 & - & - & $2-1,2-2$ & $2-1,2-2$ \\
\hline 28 & $1-1,1-2$ & $1-1,1-2$ & $1-1,1-2$ & $1-1,1-2$ \\
\hline 29 & $2-1,2-2$ & $2-1,2-2$ & $2-1,2-2$ & $2-1,2-2$ \\
\hline 31 & $\begin{array}{l}1-1,1-2, \\
3-1,3-2\end{array}$ & $\begin{array}{l}1-1,1-2 \\
3-1,3-2\end{array}$ & $\begin{array}{l}1-1,1-2, \\
3-1,3-2\end{array}$ & $1-1,1-2,3-1,3-2$ \\
\hline 33 & $\begin{array}{l}2-1,2-2, \\
3-1,3-2\end{array}$ & $\begin{array}{l}2-1,2-2 \\
3-1,3-2\end{array}$ & $\begin{array}{l}2-1,2-2, \\
3-1,3-2\end{array}$ & $2-1,2-2,3-1,3-2$ \\
\hline 35 & $1-1,1-2$ & $1-1,1-2$ & $1-1,1-2$ & $1-1,1-2$ \\
\hline 36 & $2-1,2-2$ & $2-1,2-2$ & $2-1,2-2$ & $2-1,2-2$ \\
\hline 37 & $3-1,3-2$ & $3-1,3-2$ & $3-1,3-2$ & $3-1,3-2$ \\
\hline 38 & - & - & - & $1-2$ \\
\hline 39 & $3-1,3-2$ & $3-1,3-2$ & $3-1,3-2$ & $3-1,3-2$ \\
\hline 40 & $2-1,2-2$ & $2-1,2-2$ & $2-1,2-2$ & $2-1,2-2$ \\
\hline
\end{tabular}

BW: body weight; BFW: bursa of Fabricius weight; 1-1: 1st grow-out cycle, house 1

Table 1: Schedule of samplings per grow-out cycle and according to the age of chickens

Serum samples were collected at the wing vein and kept frozen until shipment to the testing laboratory (GD Animal Health, Deventer, the Netherlands). All sera were tested using the virus neutralization test (VNT) and two enzyme-linked immunosorbent assays (ELISA): Infectious Bursal Disease Antibody Test Kit, code CK113 IBD (BioChek, Reeuwijk, the Netherlands), and Idexx IBD Ab test (Idexx, Westbrook, ME). The VNT used the protocol decribed by Skeeles et al. and by Lütticken et al. [16,17]. Briefly, fresh cultures of chicken embryo fibroblasts (CEF) were prepared. A reference IBD virus (D78 strain) was introduced at a fixed dosage of 100 tissue culture infectious doses $50 \%\left(\mathrm{TCID}_{50}\right)$; increasing dilutions of serum samples were mixed to the virus suspension. The end point of the VNT on a serum sample was determined to be the reciprocal of the highest dilution, expressed in log2, which completely neutralised 100 tissue culture infectious doses 50\% (TCID ${ }_{50}$ ) of IBDV (D78 strain), using chicken embryo fibroblasts (CEF). Due to the lack of blood sampling at hatch, it was decided later on to bleed other batches of day-old chicks born to contemporaneous parent stock flocks to obtain an approximation of the day-old IBD titres of the chicks under study. In addition, each bird was weighed, and euthanatized according to local animal welfare regulations. No other production performance data could be collected. The bursas were collected and weighed individually. This enabled us to calculate the bursa to body weight ratio (BBR), according to the formula [15]:

$B B R=$ bursa weight $(g) /$ body weight $(g) \times 100$

Each bursa was then cut in two parts: a first half was placed in a 10\%-formalin solution (10 samples per date, one tube per sample) for histopathological analysis. The formalin-fixed specimens of bursa were embedded in paraffin, sectioned ( $4 \mu \mathrm{m}$ thick) and stained with hematoxylin and eosin according to the method described by Aihara et al. [18]. The stage of the lesions indicative of IBDV replication in the bursa was read and classified under light microscopic examination. It was classified as acute, subacute, chronic inflammation or regeneration signs. Acute lesions of IBDV infection were characterized by the necrosis of bursal follicular lymphoid cells with vacuolization of cells in the medullae of follicles. Inflammation was also a prominent feature which included intrafollicular and interfollicular oedema, infiltration with variable numbers of heterophils and congestion and/or haemorrhages. Sub-acute lesions of IBDV infection were defined as the acute lesions in the bursa progressed, since the necrotic lesions, oedema and the infiltration of follicles by heterophils disappeared. The follicles became depleted from lymphocytes and replaced by reticular cells, the size of follicles was reduced. Fibroplasia of the interfollicular connective tissue and infiltration with mononuclear cells 
occurred. Chronic lesions of IBDV infection were characterized by reduction in size and number of follicles, formation of cystic follicles, marked increase in the interfollicular fibrous connective tissue and infolding of the epithelium surface of the plicae. Lastly, regeneration was indicated by the repopulation of some follicles with lymphocytes, which finally regained their original size.

The other half of the bursa was imprinted on a Whatman FTA card (GE Healthcare UK Ltd, Little Chalfont, Buckinghamshire). Five samples were imprinted per card. FTA cards and tissue samples were kept frozen for further testing using RT-PCR/RFLP analysis. A PCR analysis was done to confirm the presence of the vaccine strain (Winterfield 2512) or any field IBDV accidentally infecting the birds. Briefly, viral RNA purification from FTA card was performed by RNA rapid extraction solution and MagMax Viral RNA isolation kit according to the manufacturer's instruction. Denatured, purified RNA was used as template for the reverse transcription using MMLV-reverse transcriptase and random hexamer primers. A nested PCR method with external primers designated P1 and P2 and internal primers P2.3 and P5.3 was used [19,20]. The primers amplify a 474-base pair product spanning from 703 to 1176 nucleotides which encompasses the hypervariable region of the VP2 gene.

The bursa samples and FTA cards were processed by the same laboratory (Scientific Support Laboratory, Ceva Phylaxia, Budapest, Hungary) for histopathology and molecular tests, respectively.

\section{Statistical Analysis}

Body weight progression was modeled by quadratic gamma generalized linear models (GLM). Separate curves were fitted to the data of each cycle and house which were then tested against the restricted model that fitted a common curve to all data. The likelihood-ratio test was used. No statistical analysis was done on bursa weights or bursa to body weight ratios.

The Pearson correlation between the different serology test methods was calculated using the average titres obtained in a given cycle and house on a given day. The likelihood ratio test was used to compare two linear models run on the standardized data of titres: the full model contained individual linear regressors for each cycle and house pair while the restricted model contained a shared regressor for all pairs.

All tests were evaluated at $95 \%$ confidence level $(\mathrm{p}<0.05)$.

\section{Results and Discussion}

\section{Body Weight}

Average body weights of 1,754 to $1,883 \mathrm{~g}$ (grow-out cycle 1), 1,984 to 2,190 g (cycle 2), and 2,333 to 2,413 g (cycle 3) were reached at study completion, i.e. $\mathrm{d} 35, \mathrm{~d} 40$, and $\mathrm{d} 39$, respectively (Figure 1). The body weight standard of the corresponding genetic line (Ross 308 Broiler Performance Objectives, 2014 Aviagen) is included as reference.

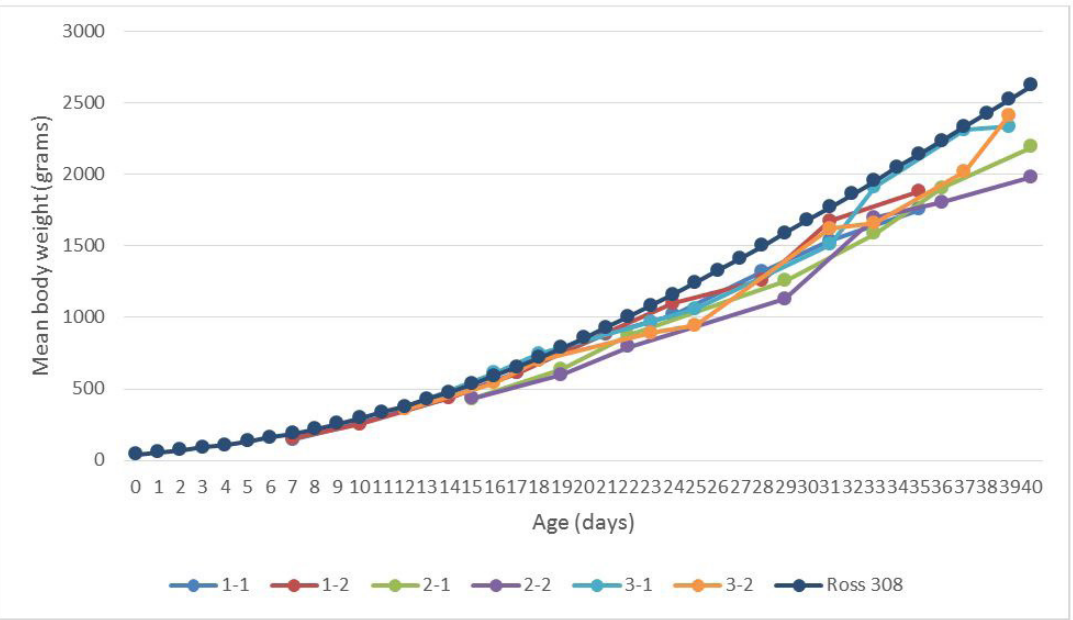

Figure 1: Mean body weight (in grams) of commercial broilers (sex as hatched) in the six tested flocks (cycle 1 [1-1, 1-2], cycle 2 [2-1, 2-2], cycle 3 [3-1, 3-2]). All of them were in ovo vaccinated using an immunecomplex vaccine against IBD in the hatchery. The genetic line's body weight standard is included (Ross 308)

According to the likelihood-ratio test the observed difference in body weights between cycles and houses was not greater than expected by chance, thus it was justified to fit a common quadratic curve to the pooled data of all cycles and houses. No statistically significant difference could be shown when analyzing the body weights curves of the cycles and the houses ( $\mathrm{p}=0.9988$, likelihoodratio test). Therefore, the observed differences between the cycles/houses were explained by the pure variance of the animals and were not attributable to a cycle effect. To compare the vaccinated flocks' average body weight to the breed's standards, the 95\% confidence band of the fitted curve was calculated to check if the breed's standards figures lie outside it. Since the standard lies outside the confidence interval, the average body weight of the vaccinated flocks was significantly different from the standard (data not shown). It is noteworthy that a low average body weight was recorded from the first sampling onwards, i.e. as early as 7, 15, and 12 days of age during cycles 1,2, and 3, respectively. For instance, in cycle 1, average body weights of 7-day old broilers were approximately $20 \%$ lighter than the Ross 308 standard. No investigation was made to definitely attribute this poor average weight to brooding procedures, feed, or chick quality, among other possible causes. This early growth delay was probably critical 
in explaining the overall body weight deficiency at the end of rearing, because the 7-day weight has a direct influence on the final weight [21]. This delay was consistently recorded throughout the study in the six flocks; therefore, it could be likely due to poor management issues of the farm, or more generally, of the producing company. The necropsy analyses at sampling did not provide clear information about any disease challenge. No definite conclusion could be drawn, however, since other performance indicators, like feed efficiency, condemnation rates at slaughterhouse, or overall mortality rate, were not provided by the producer.

\section{Bursa Appearance and Weight}

Swelling of bursa was rarely detected: it was observed in $20 \%$ of chickens from both houses ( $1^{\text {st }}$ grow-out cycle) at 31 days; it was observed at 27 days of age in 10 and $20 \%$ of chickens from flocks 2-1 and 2-2, respectively, and in $20 \%$ of chickens from both houses at 29 days of age. No bursa oedema was recorded from any sampled bird during the third cycle (both flocks). The close monitoring of bursa weight is a way to monitor the success of the vaccine's administration, since it is the main site for IBDV replication [14]. Following a steady growth, the mean bursa weight reached a peak of 2.3 and $2.0 \mathrm{~g}$ by d28 in first, of 1.6 by d22 and $1.7 \mathrm{~g}$ by d29 in second, and of 1.5 and $1.7 \mathrm{~g}$ by d18 in third broiler cycles, respectively (Figure 2). Afterwards, a decrease in mean bursa weight was observed until the $5^{\text {th }}$ week of age, and the bursas became lighter (averaging $1.0 \mathrm{~g}$ ). In the subsequent weighing ( $\mathrm{d} 40$ and $\mathrm{d} 39$ in the $2^{\text {nd }}$ and $3^{\text {rd }}$ cycles, respectively), the bursa weight remained stable.

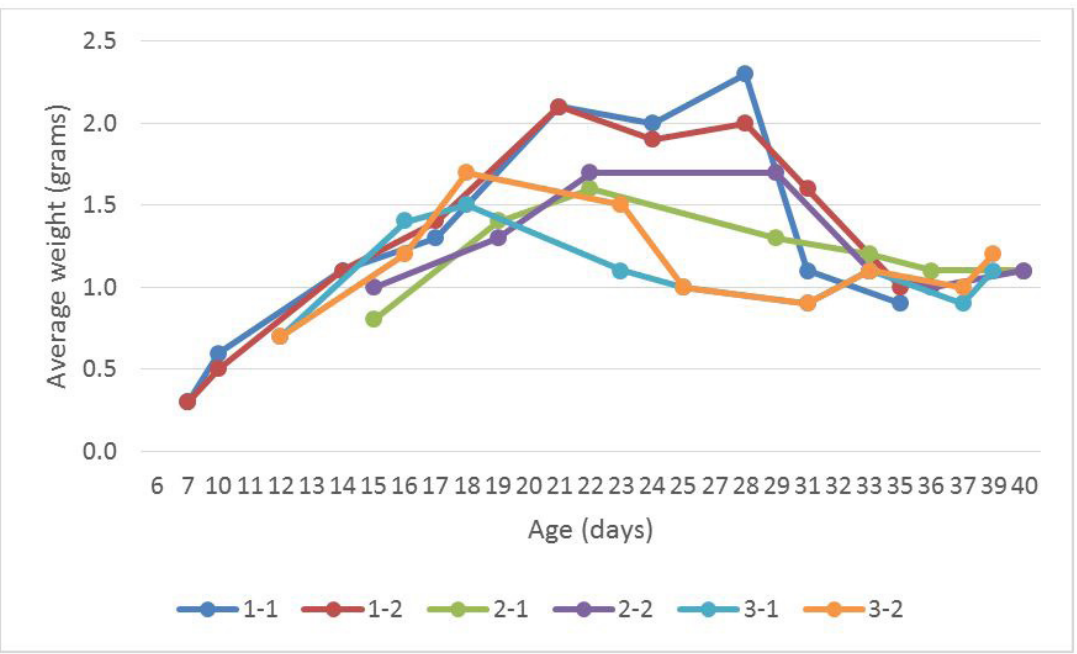

Figure 2: Mean bursa weight (in grams) of commercial broilers (sex as hatched) in the six tested flocks (cycle $1[1-1,1-2]$, cycle 2 [2-1, 2-2], cycle 3 [3-1, 3-2]). All of them were in ovo vaccinated using an immune-complex vaccine against IBD in the hatchery

\section{BB Ratio}

Besides body and bursa weight, the ratio of the bursa weight to the body weight (BBR) is an additional indicator which is commonly used in the scientific literature. They are shown in (Figure 3). The calculated BBR was stable between 0.20 to 0.24 in the six flocks from the beginning of sampling until approximately 3 weeks of age: it reached a maximum figure of 0.24 at d21, of 0.22 at d19, and of 0.23 to 0.24 at $\mathrm{d} 16$ to d18 in samples coming from cycles 1,2, and 3, respectively. Afterwards, the BBR paralleled the drop in bursa weight, since it reached eventually a figure of 0.05 at d35, of 0.05 and 0.06 at $\mathrm{d} 40$, and of 0.05 at $\mathrm{d} 39$ in samples coming from cycles 1,2 , and 3 , respectively.

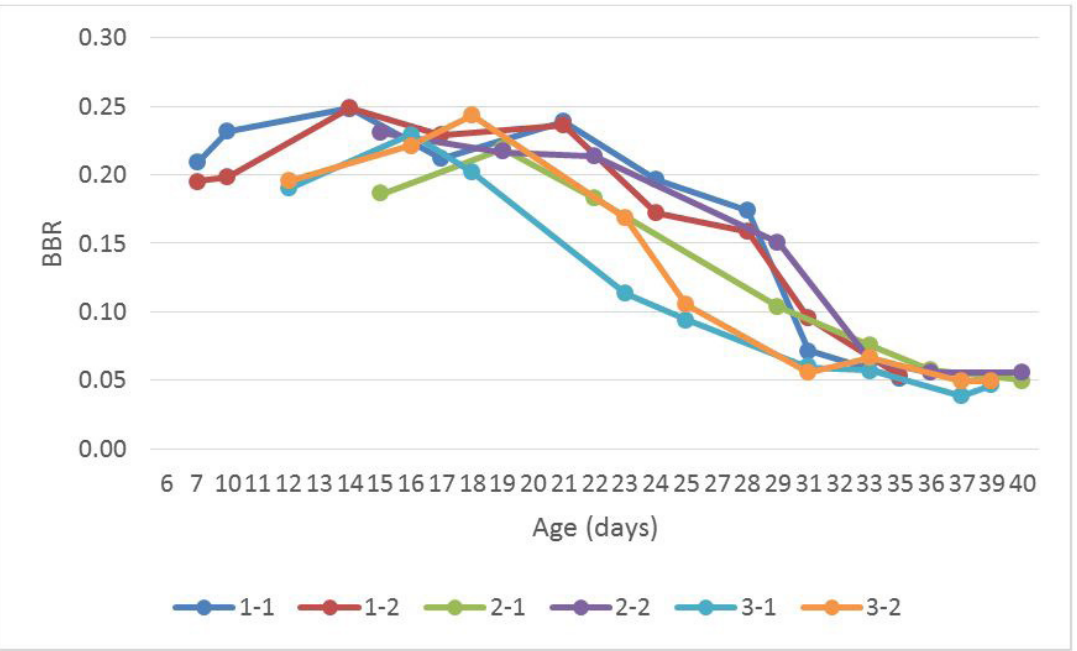

Figure 3: Bursa to body weight ratio of commercial broilers (sex as hatched) in the six tested flocks (cycle $1[1-1,1-2]$, cycle $2[2-1,2-2]$, cycle $3[3-1,3-2])$. All of them were in ovo vaccinated using an immunecomplex vaccine against IBD in the hatchery 
Cazaban et al. proposed a bursa to body weight ratio (BBR) standard of at least 0.11 in male Cobb 500 broilers that were raised in the absence of pathologic conditions and vaccination [22]. No conclusion could be drawn about the obtained BBR in the present study (0.04 and above), since it involved vaccinated and unsexed Ross 308 broilers. Therefore, further studies are warranted to set up a similar BBR standard in Ross 308 chickens, with and without vaccination.

\section{Histopathology and PCR Results}

The six tested flocks showed similar patterns of bursa inflammatory process at histological analysis and of onset of vaccine virus sequence detection by PCR. Flock 3-2 was chosen as representative of the other ones and to avoid redundancy. Figure 4 shows the schedule and quick outcome of bursa lesions as per histopathological analysis (acute, subacute, chronic, regeneration signs [abbreviated as "regen"]), together with the result of the RT-PCR and RFLP analysis to identify the detected IBD virus. Virus sequence detection rates by molecular method (RT-PCR) correlated well with histopathological findings, i.e. any bursa showing histological lesions indicative of IBDV replication were found positive by PCR as well. The Winterfied 2512 strain of the vaccine was the only IBD virus sequence detected in the samples. PCR test was positive for vaccine virus sequence from 18 days of age onwards, whereas histopathological analysis detected first changes from 23 days of age onwards. The impact of these findings on the overall state of health of the vaccinated chickens was non-existent in this trial. Additional helpful information was provided by another study. A bursectomy (surgical removal of the bursa without killing birds) trial showed that beyond two weeks of age, the existence of bursa of Fabricius was not critical to the antibody response: 2-week old (and older) surgically bursectomized chickens were vaccinated using a live Newcastle disease (ND) vaccine to assess whether they were still immunocompetent or not: they showed a similar onset and intensity of post-vaccination antibody response than control chickens did with an intact bursa [23]. When the surgical removal of the bursa was done prior to 2 weeks of age, some degree of immune suppression could be observed, since antibody response to ND vaccination was significantly lower than in controls. This result was in agreement with previous experiments [24]. In the present study, bursa lesions (inflammation and atrophy) as a result of vaccine virus replication occurred later than 3 weeks of age, regardless of the vaccinated flock; in addition, they were of short duration until recovery (one week approximately). As a result, the lack of any risk was confirmed.
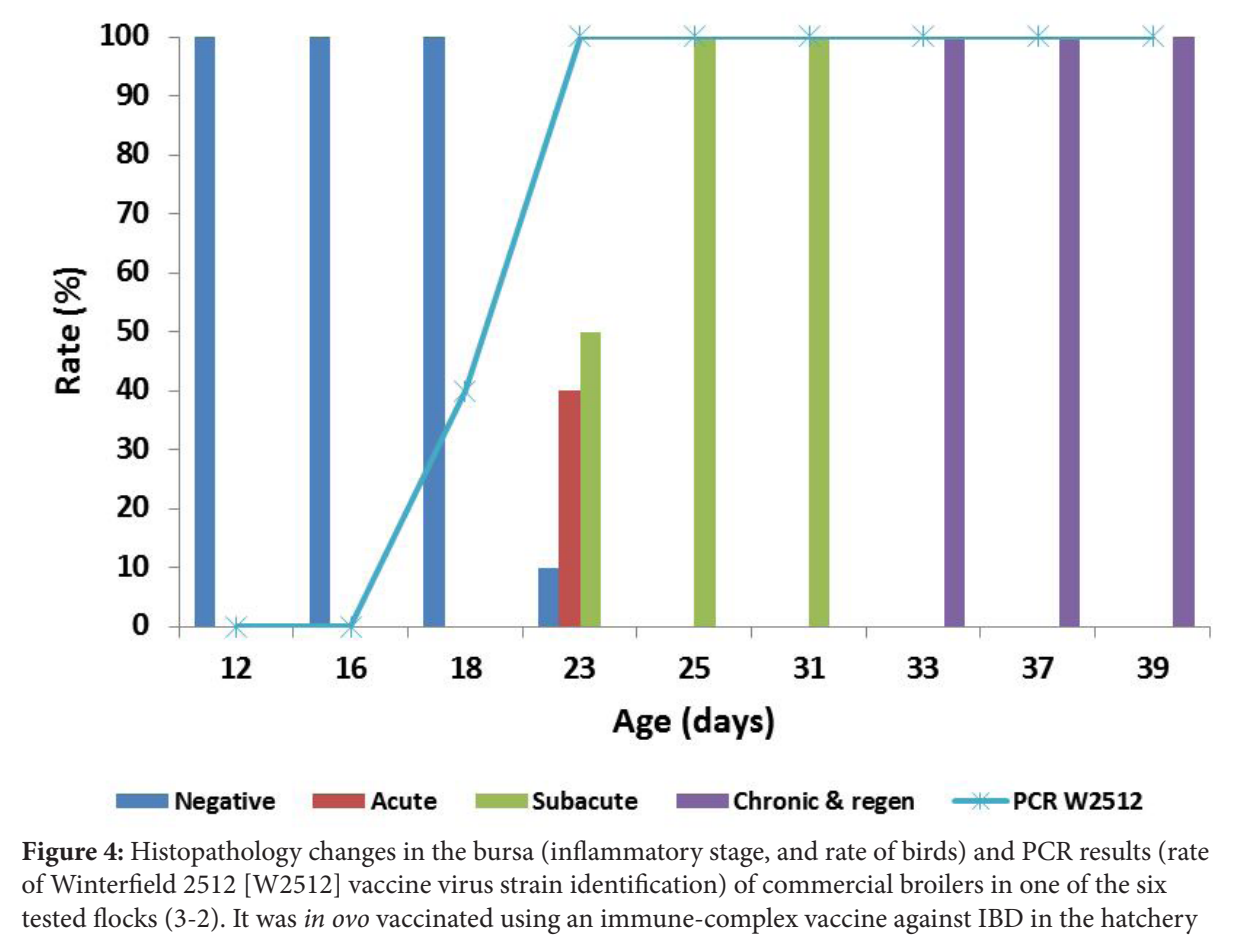

\section{Serology}

Results are presented in (Figures 5,6 and 7). The six tested flocks showed a similar low serology profile at start, regardless of the technique used. By Idexx ELISA the starting antibody titres were 911 and 377 at 10 days of age (first cycle), 288 and 293 at 15 days of age (second cycle), and 310 and 193 at 12 days of age (third cycle). By BioChek ELISA (Figure 6), the titres in the same ages were 979 and 667 (first cycle), 283 and 383 (second cycle), and 504 and 300 (third cycle) (Figure 5). Antibody titres declined further or remained as low as at start until 22-28 or 18-22 days of age using Idexx or BioChek ELISA kits, respectively. Afterwards, they started increasing, indicating the development of an active immune response. BioChek ELISA kit indicated the development of an active immune response sooner and with a sharper titre rise than the Idexx kit on the same serum samples. BioChek kit displayed higher titres than Idexx kit for the same set of serum samples. This is consistent with previous findings [25]. Virus neutralization 
titres showed a similar profile of declining and then increasing trends, but with much fewer variations compared to the ELISA titres (VN, Figure 7). The starting VN antibody titres were $10 \log 2$ at 10 days of age (first cycle), 8 and $11 \log 2$ at 15 days of age (second cycle), and 9 and $10 \log 2$ at 12 days of age (third cycle). VN titres reached the lowest figure of 5 log2 in only two instances (by $\mathrm{d} 22$ in 2-1, and by d18 in 3-1 flocks, respectively). Virus neutralization test was carried out because it is generally regarded as the reference for the assessment of the immunity to Gumboro disease [1]. A cut off of $3 \log 2$ (i.e. a titre of 8 ) is generally accepted: any VN titre above it is regarded as positive [26]. Other authors rather suggested $3.3 \log 2$ (or a titre of 10) as VN positivity threshold [27]. A good correlation was found between VN titre and protection against challenge [11,27]. However, these two publications assessed the protection supported by the passive immunity (MDA) in SPF (specific pathogen free) chickens only. Therefore, their conclusions cannot be directly extrapolated to the active immunity in commercial broilers in field conditions. To the best of the authors' knowledge, a protective VN titre standard of active immunity in commercial broilers against challenge is lacking.

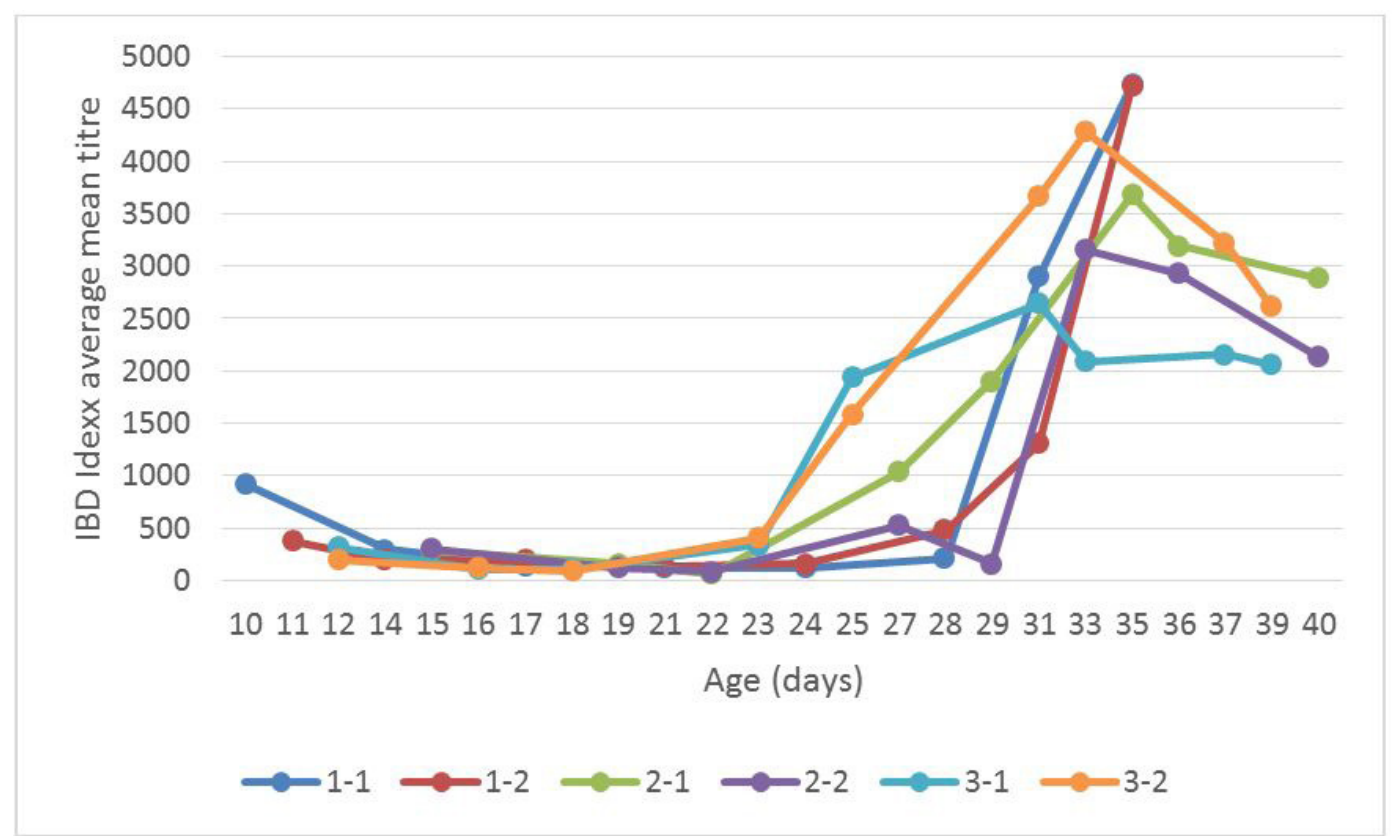

Figure 5: IBD serology (Idexx ELISA) of commercial broilers in the six tested flocks (cycle 1 [1-1, 1-2], cycle 2 [2-1, 2-2], cycle $3[3-1,3-2])$. All of them were in ovo vaccinated using an immune-complex vaccine against IBD in the hatchery

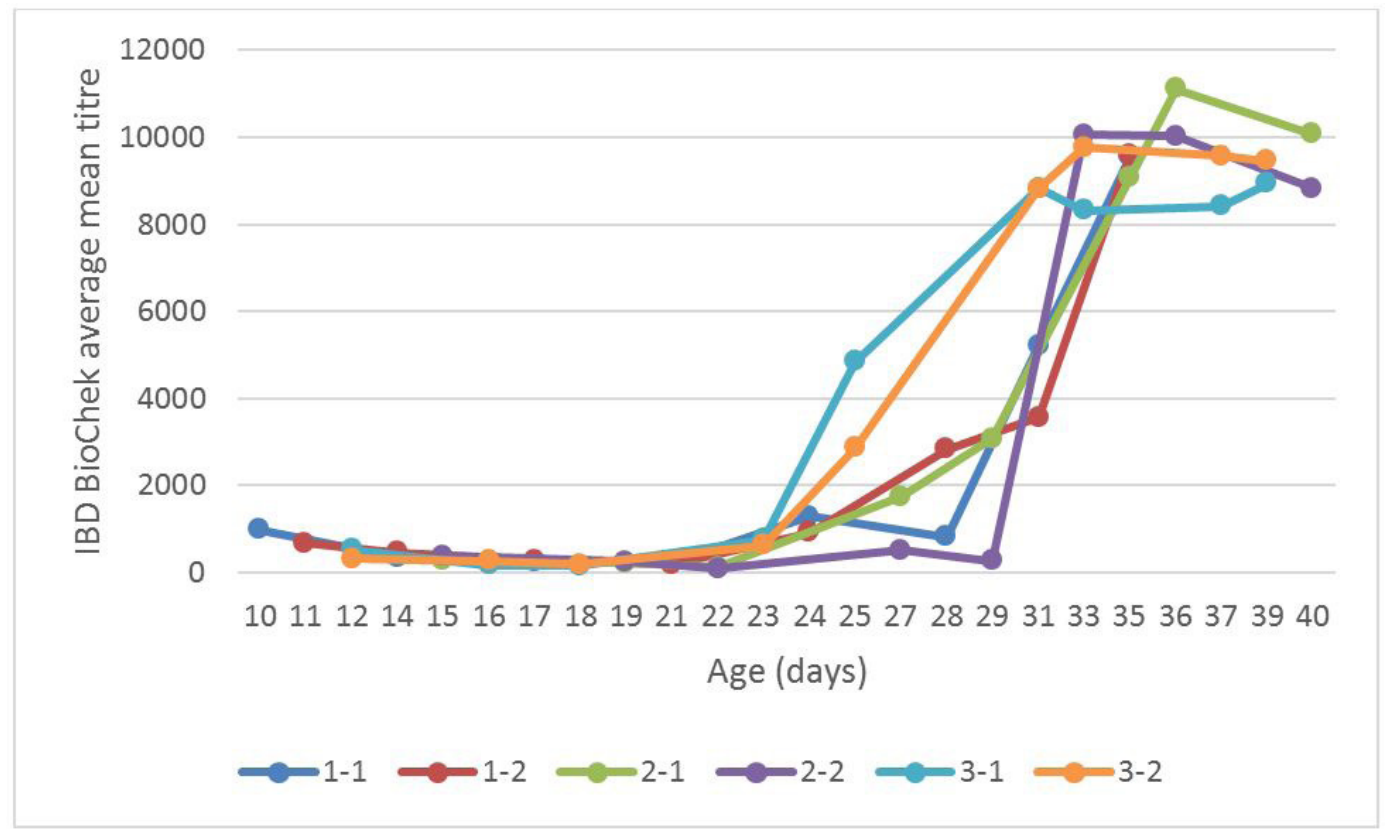

Figure 6: IBD serology (BioChek ELISA) of commercial broilers in the six tested flocks (cycle 1 [1-1, 1-2], cycle 2 [2-1, 2-2], cycle $3[3-1,3-2]$ ). All of them were in ovo vaccinated using an immune-complex vaccine against IBD in the hatchery 


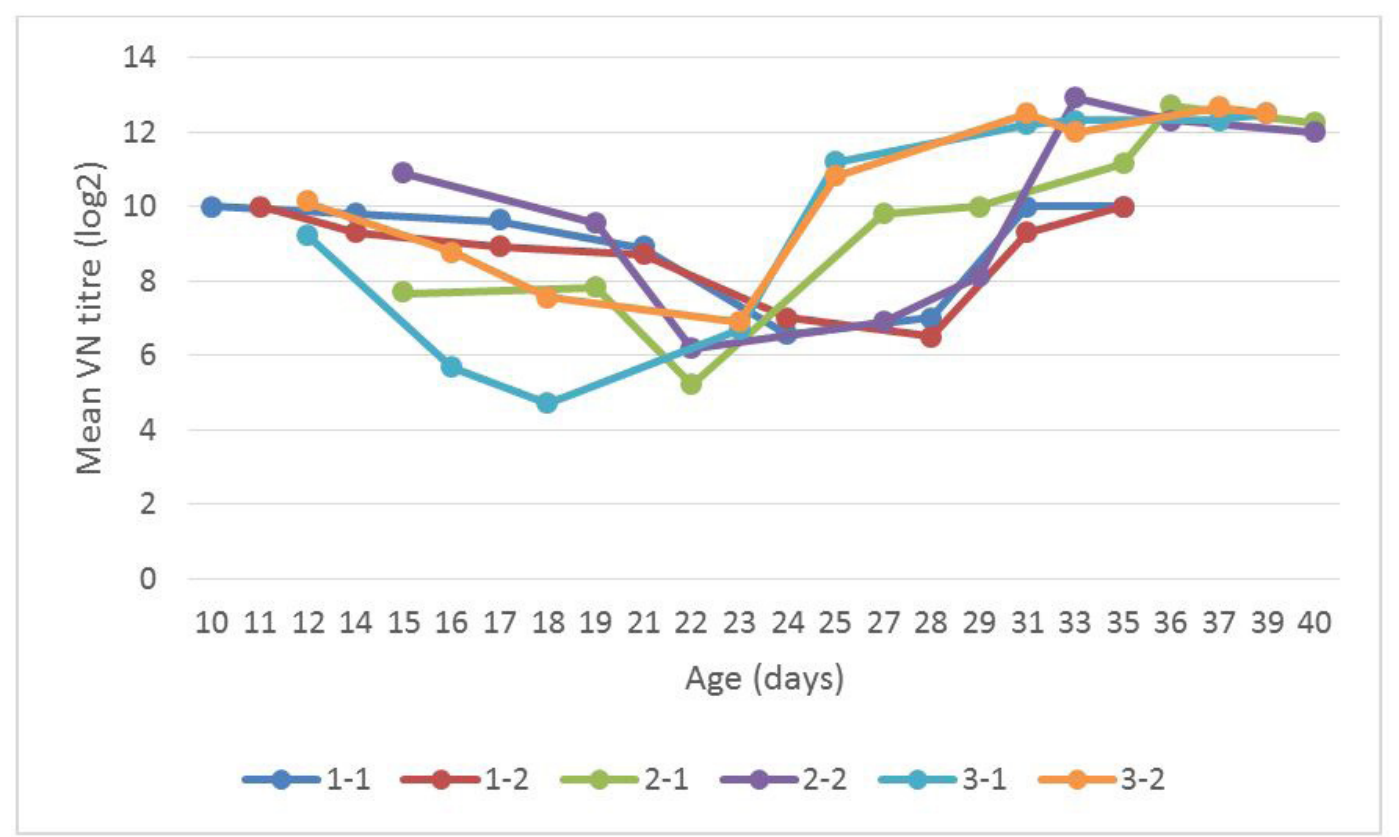

Figure 7: IBD serology (virus neutralization test) of commercial broilers in the six tested flocks (cycle 1 [1-1, 1-2], cycle 2 [2$1,2-2]$, cycle 3 [3-1, 3-2]). All of them were in ovo vaccinated using an immune-complex vaccine against IBD in the hatchery

The seropositivity rate among flocks was assessed throughout the study (data not shown), according to the cut-off value that is provided by the ELISA kit manufacturers (396 in Idexx, 391 in BioChek), or according to the cut-off of the VN test (3.2 log2). Only a few early blood samplings were still seropositive by ELISA: only the average titre of flock 1-1 at d10 was seropositive by Idexx ELISA (911). According to BioChek ELISA, seropositivity was shown in the two flocks of the first cycle at d10 (979, 667), and in one of the two flocks of the third cycle at d12 (504). The first samples of the two flocks of the second cycle (d15) were already seronegative. The seropositivity rate dropped and remained negative in all tested flocks for 11 days (from 11 to 22 days of age) or for 7 days (from 15 to 22 days of age) when using Idexx or BioChek ELISA kits, respectively. Between 23 and 29 days of age, $50 \%$ to $100 \%$ of the broiler flocks were ELISA seropositive using Idexx kit, whereas beyond 29 days of age, 100\% were seropositive. When using BioChek kit, however, all flocks became seropositive from d23 onwards (except a mean titre of 258 that was obtained by d29 in flock 2-2). On the contrary, VN testing results showed a consistent $100 \%$ seropositivity rate in all broiler flocks throughout the entire duration of the study. Contrary to ELISA, VN test detected the presence of residual MDA till the point where the active immune response started. By VN test, there was no antibody negativity 'window', as was experienced when ELISA was used. This study confirmed that VN antibodies could be detected, in a time frame when ELISA showed negative results, as already demonstrated by others [26]. It showed that these two tests detected different types of antibodies and had a different sensitivity. VN titres declined as MDA were metabolized, and then increased again as evidence of active immunity. The lowest recorded VN titres $(5 \log 2)$ were observed at around 18 or 22 days of age; afterwards, VN titres increased again to eventually reach 12 to 13 $\log 2$ at study completion. These VN results were consistent with previous findings, where Cevac ${ }^{\circledast}$ Transmune vaccine take was demonstrated in presence of a residual MDA titre of $4.9 \log 2$ [28]. At that time, the chickens were still protected by MDA against a very virulent (vv) IBDV challenge.

The Pearson correlation between the different methods was calculated using the average titres obtained in a given cycle and house on a given day. As there was some variability between the titres obtained in different grow-out cycles and houses, the first step was to assess whether the correlation between the methods significantly varied between cycles and houses. The likelihood ratio test was used to compare two linear models run on the standardized data of titres: the full model contained individual linear regressors for each cycle and house pair while the restricted model contained a shared regressor for all pairs. The likelihood-ratio test was nonsignificant in any of the three cases (Idexx - BioChek ELISA, Idexx ELISA - VN and BioChek ELISA - VN), therefore the Pearson correlation coefficients between Idexx and BioChek ELISA, between Idexx ELISA and VN and between BioChek ELISA and VN were not significantly different across cycles and houses. As a consequence, the data from different cycles and houses could be pooled and the Pearson correlation could be estimated from the pooled dataset. The highest correlation was found between Idexx and BioChek ELISA (0.93), and the lowest correlation was found between Idexx ELISA and VN (0.68), whereas BioChek ELISA and $\mathrm{VN}$ showed a correlation of 0.78 . Further studies involving more serum samples are warranted to confirm or amend these correlation coefficients.

No day-old blood collection was done in the actual flocks under test. The earliest blood samples were collected at 10 days of age (flock 1-1). The mean ELISA titre was close to 1000 (911 using Idexx, 979 using BioChek). The half-life of MDA directed against IBDV and as detected by commercial ELISA kits in broilers is generally considered to be approximately 3 to 3.5 days [29]. As a 
consequence, a reverse calculation would indicate a theoretical mean Idexx ELISA titre of 1822 by 7 days of age, and of 3644 by 4 to 0 days of age. MDA titre is stable for the first three days of life, since the antibody release from egg yolk in the first 3 days of life compensates the first stages of catabolism; therefore, this figure could be regarded as relevant to assess the actual day-old titre of the chicks under study. This figure (3644) could be considered moderate to low. It is consistent with the serology that was performed in day-old chicks that were hatched to other parent stocks of similar age (Table 2): at hatch, day-old chicks showed a mean Idexx Elisa titre between 3122 and 4395, depending on the chicks' batch. In the same way, the measured VN titres in the present study were on average $10 \log 2$ at around 10 days of age (i.e. a titre of 1024). By calculating backwards using the half-life of MDA (3.5 days), day-old titres would have approached $12 \log 2$, i.e. a value of 4096 . Regardless of the serology test, it is fair to assume that the day-old MDA titres of the chicks in this study were probably inferior, or close to 4000.

\begin{tabular}{|c|c|c|c|c|}
\hline $\begin{array}{c}\text { Trial's DOC flock } \\
\text { No. }\end{array}$ & $\begin{array}{c}\text { PS age of trial's } \\
\text { DOC flocks }\end{array}$ & $\begin{array}{c}\text { Other PS flocks of } \\
\text { similar age }\end{array}$ & $\begin{array}{c}\text { Mean IBD titre in } \\
\text { DOC }\end{array}$ & CV \\
\hline $1-1,1-2$ & 32 & 33 & 3324 & $64 \%$ \\
\hline & & 35 & 3122 & $42 \%$ \\
\hline $2-1,2-2$ & 42 & 43 & 4395 & $49 \%$ \\
\hline $3-1,3-2$ & 44 & 43 & 4198 & $48 \%$ \\
\hline
\end{tabular}

Table 2: Mean IBD Idexx ELISA titre and coefficient of variation (CV) in day-old chicks (DOC) from similar parent stock (PS) flocks of the same company

A similar field monitoring of the same immune-complex IBD vaccine with short sampling intervals was previously conducted in France in standard broilers, with a level of MDA at hatch between 4000 and 5000 using BioChek ELISA [30]. Interestingly, very similar findings were observed in that study, compared to the present one: a transient inflammation in bursa tissues, a vaccine virus detection by molecular methods and a similar pattern of antibody response. This is further confirming the consistency of the mechanism of action of this vaccine technology. In addition, vaccinated chicks with a "medium" (4000 to 5000) or a "low" (less than 4000, [the present study]) antibody titre at hatch showed a similar successful immunization process [30].

\section{Conclusions}

In conclusion, chicks with relatively low MDA levels $(<4000)$ were successfully immunized using the immune-complex IBD vaccine under field conditions with no detectable negative effect. Evidences were provided by the expected performances and outcome of vaccine mechanism of action, namely (i) invading the bursa of Fabricius, (ii) inducing a transient atrophy and inflammation until tissue recovery, (iii) and inducing antibody response as a last step. Idexx and BioChek ELISA kits were able to detect MDA disappearance as well as the onset of active immunity, but both were unable to detect antibodies for a given period of time due to their respective cut off values. Conversely, VN test was able to detect antibodies more consistently for the whole duration of the study. VN monitoring also showed a longer persistence of MDA than shown when using ELISA test. This provides evidence that every test has its strengths and weaknesses. It is therefore important to use a comprehensive set of investigation tools in order to conduct meaningful field monitoring or diagnostic surveillance.

\section{Acknowledgement}

The authors would like to thank Krisztian Kertesz for the statistical analyses.

\section{References}

1. Glisson JR, McDougald LR, Nolan LK, Suarez DL, Nair VL (2013) Infectious bursal disease In: Diseases of Poultry (13 ${ }^{\text {th }}$ Edn) Ames: Iowa State Press, USA.

2. Hernandez M, Tomas G, Marandino A, Iraola G, Maya L, et al. (2015) Genetic characterization of South American infectious bursal disease virus reveals the existence of a distinct worldwide-spread genetic lineage. Avian Pathol 44: 212-21.

3. Eregae ME, Dewey CA, McEwen SA, Ouckama R, Ojkic D, et al. (2014) Flock prevalence of exposure to avian adeno-associated virus, chicken anemia virus, fowl adenovirus, and infectious bursal disease virus among Ontario broiler chicken flocks. Avian Dis 58: 71-7.

4. Jackwood DJ, Stoute ST (2013) Molecular evidence for a geographically restricted population of infectious bursal disease viruses. Avian Dis 57: 57-64.

5. Kasanga CJ (2015) Molecular typing of infectious bursal disease virus field strains in endemic settings of Africa. Res Opin Ani Vet Sci 5: 429-34.

6. Li K, Courtillon C, Guionie O, Allée C, Amelot M, et al. (2015) Genetic, antigenic and pathogenic characterization of four infectious bursal disease virus isolates from China suggests continued evolution of very virulent viruses. Infect Genet Evol 30: 120-7.

7. Amin OGM, Jackwood DJ (2014) Identification and molecular analysis of infectious bursal disease in broiler farms in the Kurdistan Regional Government of Iraq. Trop Anim Health Prod 46: 1297-301.

8. Mawgod SA, Arafa AS, Hussein HA (2014) Molecular genotyping of the infectious bursal disease virus (IBDV) isolated from broiler flocks in Egypt. International Journal of Veterinary Science and Medicine 2: 46-52.

9. Bonci M, Giovanardi D, Pesente P, Morandini E, Lupini C, et al. (2013) Molecular characterization of infectious bursitis virus strains isolated recently in Italy [Caratterizzazione molecolare di ceppi del virus della bursite infettiva isolati recentemente in Italia]. Atti della Società Italiana di Patologia Aviare 136-41.

10. Cortey M, Bertran K, Toskano J, Majo N, Dolz R (2012) Phylogeographic distribution of very virulent infectious bursal disease virus isolates in the Iberian Peninsula. Avian Pathol 41: 277-84. 
11. Maas RA, Venema S, Oei HL, Pol JMA, Claassen IJTM, et al. (2001) Efficacy of inactivated infectious bursal disease (IBD) vaccines: comparison of serology with protection of progeny chickens against IBD virus strains of varying virulence. Avian Pathol 30: 345-54.

12. Comte S, Alva B, Paulet P (2013) The global evolution of new technology vaccines for use in the hatchery. International Hatchery Practice 27: 23.

13. Jacquinet C, Gardin Y (2011) Monitoring the vaccine intake of a Gumboro vaccine of complex immune type applied to the hatchery. Interest and examples of follow-up in comparison with a traditional vaccination method [Monitoring de la prise vaccinale d'un vaccin Gumboro de type immune complexe appliqué au couvoir. Intérêt et exemples de suivis en comparaison avec une méthode de vaccination classique]. Proceedings of the Journées de Recherche Avicole, Tours, France.

14. Kelemen M, Forgach K, Ivan J, Palya V, Suveges T, et al. (2000) Pathological and immunological study of an in ovo complex vaccine against infectious bursal disease. Acta Vet Hung 48: 443-54.

15. Darteil R, Bublot M, Laplace E, Bouquet JF, Audonnet JC, et al. (1995) Herpesvirus of turkey recombinant viruses expressing infectious bursal disease virus (IBDV) VP2 immunogen induce protection against an IBDV virulent challenge in chickens. Virology 211: 481-90.

16. Skeeles J, Lukert P, De Buysscher E, Fletcher O, Brown J (1979) Infectious Bursal Disease Viral Infections. I. Complement and Virus-Neutralizing Antibody Response Following Infection of Susceptible Chickens. Avian Dis 23: 95-106.

17. Lütticken D, Cornelissen DRW (1981) Plaque reduction test and microneutralization test for the detection of neutralizing antibodies to the virus of infectious bursitis (IBDV) [Plaquereductionstest und Mikroneutralisationstest zum Nachweis neutralisierender Antikörper gegen das Virus der infectiösen Bursitis (IBDV)]. Dtsch Tierartzl Wochenschr 88: 506-8.

18. Aihara N, Horiuchi N, Hikichi N, Ochiai M, Hosoda Y, et al. (2015) Immunoreactivity and morphological changes of bursal follicles in chickens infected with vaccine or wild-type strains of the infectious bursal disease virus. J Vet Med Sci 77: 913-8.

19. Lin Z, Kato A, Otaki Y, Nakamura T, Sasmaz E, et al. (1993) Sequence comparisons of a highly virulent infectious bursal disease virus prevalent in Japan. Avian Dis $37: 315-23$.

20. Liu HJ, Giambrone JJ, Dormitorio T (1994) Detection of genetic variations in serotype I isolates of infectious bursal disease virus using polymerase chain reaction and restriction endonuclease analysis. J Virol Methods 48: 281-91.

21. Laughlin K (2005) The importance of a good start in life. International Poultry Production 13: 13-4.

22. Cazaban C, Majo Masferrer N, Dolz Pascual R, Nofrarias Espadamala M, Costa T, et al. (2015) Proposed bursa of Fabricius weight to body weight ratio standard in commercial broilers. Poult Sci 94: 2088-93.

23. Palya V, Walkóné Kovács E, Felföldi B, Gardin Y (2015) Assesment of humoral immune response to live Newcastle disease (ND) vaccination in surgically bursectomized broiler chickens. Proceedings of the XIXth International Congress of the World Veterinary Poultry Association, Cape Town, South Africa.

24. Faragher JT, Allan WH, Wyeth PJ (1974) Immunosuppressive effect of infectious bursal agent on vaccination against Newcastle disease. Vet Rec 95: 385-8.

25. Gardin Y, Palya V, Sesti L, Moore Dorsey K (2008) Efficacy of infectious bursal disease virus vaccine against various forms of the disease. Proceedings of the American Association of Avian Pathologists annual meeting, New Orleans, LA.

26. Rautenschlein S, Kraemer C, Vanmarcke J, Montiel E (2005) Protective efficacy of intermediate and intermediate plus infectious bursal disease virus (IBDV) vaccines against very virulent IBDV in commercial broilers. Avian Dis 49: 231-7.

27. Lucio B, Hitchner SB (1979) Infectious bursal disease emulsified vaccine: Effect upon neutralizing-antibody levels in the dam and subsequent protection of the progeny. Avian Dis 23: 466-48.

28. Herczeg J, Nagy M, Makranszki L, Balla E, Kustos K, et al. (2011) Laboratory efficacy testing of subcutaneously administered Cevac Transmune vaccine in broiler chickens. Proceedings of the XVIIth International Congress of the World Veterinary Poultry Association, Cancun, Mexico.

29. De Wit JJ (1998) Gumboro disease: estimation of optimal time of vaccination by the Deventer formula. Pol Vet J 3: 19-22.

30. Laboute M, Albaric O, Cazaban C, Palya V, Alva B, et al. (2013) Kinetics of the development of immunity following the vaccination with an infectious bursal disease immune-complex vaccine. Proceedings of the XVIIIth International Congress of the World Veterinary Poultry Association, Nantes, France.

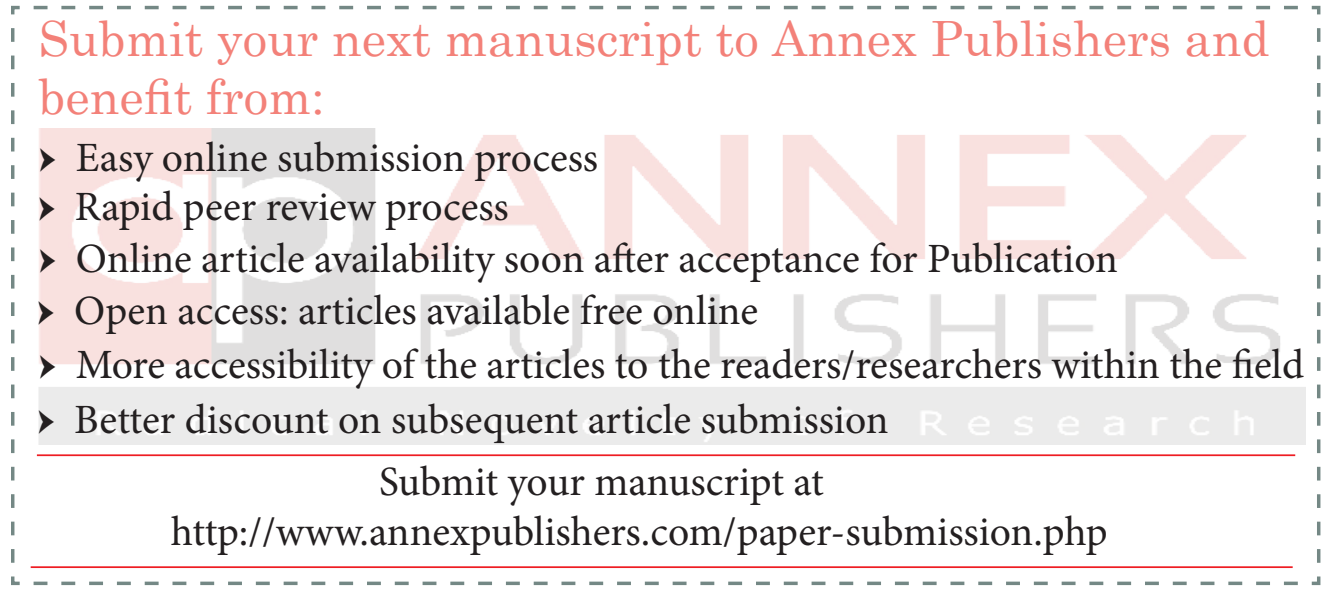

